\title{
Positive association between artificially sweetened beverage consumption and incidence of diabetes. Reply to Sylvetsky Meni AC, Swithers SE, Rother KI [letter]
}

\author{
Fumiaki Imamura $^{1}$ - Laura O'Connor ${ }^{1}$ - Nita G. Forouhi ${ }^{1}$ \\ Received: 25 June 2015 / Accepted: 30 June 2015 / Published online: 24 July 2015 \\ (C) Springer-Verlag Berlin Heidelberg 2015
}

Keywords Artificially-sweetened beverages .

Epidemiology $\cdot$ Sugar-sweetened beverages $\cdot$ Type 2 diabetes

\begin{abstract}
Abbreviations
ASB Artificially sweetened beverages

SSB Sugar-sweetened beverages
\end{abstract}

To the Editor: We appreciate the positive comments from Sylvetsky Meni et al [1] about our work on consumption of sweet beverages and incidence of type 2 diabetes [2]. However, they question our interpretation of reverse causality as the possible explanation for the attenuation of the significant association between consumption of artificially sweetened beverages (ASB) and incidence of diabetes after adjustment for obesity, proposing that alternative explanations are more likely. In particular, they argue for a direct detrimental effect of artificial sweeteners on metabolic health, rather than our rationale that obese individuals tend to consume ASB preferentially as part of weight management strategies. They also suggest that our approach of adjusting for BMI in our analysis of ASB was inappropriate, because consumption of ASB causes greater adiposity and insulin resistance.

Sylvetsky Meni et al cite several studies to support the argument that artificial sweeteners may have direct adverse

Nita G. Forouhi

Nita.Forouhi@mrc-epid.cam.ac.uk

1 Medical Research Council Epidemiology Unit, University of Cambridge School of Clinical Medicine, Box 285 Institute of Metabolic Science, Cambridge Biomedical Campus,

Cambridge CB2 0QQ, UK metabolic effects. However, the evidence is far from conclusive. For example, the dose of saccharin used in experiments in rats [3] and in seven human volunteers [4] was in supraphysiological doses, several-fold higher than typical human consumption of saccharin ( $8 \mathrm{mg}$ per $340 \mathrm{ml}$ or $12 \mathrm{oz}$ ) [5]. We estimate that approximately 40 cans/day of a $12 \mathrm{oz} \mathrm{ASB}$ drink with saccharin would have to be consumed to achieve that daily dose of saccharin. Their citation of studies of a pancreatic beta cell line [6] or of 17 morbidly obese adults with mean BMI of $41.0 \mathrm{~kg} / \mathrm{m}^{2}$ [7] have limited generalisability, and such assertions should be tested for comparison with observations, including ours, in a free-living setting. Moreover, the observational studies from a single cohort $[8,9]$ cited by Sylvetsky Meni et al did not show any causal effects of ASB on increased body weight or adiposity, being limited by the possibility that individuals with greater weight or weight gain might have preferentially consumed ASB or that ASB consumers may have consumed food with a high energy content to compensate for a low energy intake by consuming ASB.

In contrast, though not cited by Sylvetsky Meni et al, there is convincing evidence from randomised controlled trials that ASB can play an important part in weight control and obesity prevention. For example, Maersk et al reported a 6 month intervention study comparing water, milk, sugar-sweetened beverages (SSB), and ASB sweetened with aspartame [10]. Among 47 adults, SSB consumers showed trends toward greater fat mass in adipose tissue, muscle and liver than other groups, whereas there was no difference in fat mass in adipose, muscle or liver tissues when comparing ASB consumers with consumers of water or milk. Tate et al conducted a 6 month trial ( $n=318$ overweight and obese individuals) comparing groups of no intervention, substituting water for SSB, and substituting ASB for SSB, and demonstrated significant weight loss by the groups that consumed ASB or water, and reported no difference between ASB and water consumption 
[11]. These trials indicate that an obesogenic effect of ASB is absent or negligible. Moreover, although Sylvetsky Meni et al express concern about the increasing use of diet and lowenergy beverages in place of SSB among children, trial evidence indicates reduced weight gain and lower fat accumulation with such replacement [12]. Given the trial evidence, a correlation between ASB consumption and obesity status is likely to reflect that obesity status alters ASB consumption. Although no trial to date has randomised participants by obesity status or health consciousness and examined behavioural changes, we consider that our approach of adjusting for obesity status in analysis of ASB is valid. Without the adjustment, the result would reflect a portion of the established effect of obesity on the development of diabetes. Indeed, we deliberately provided our results both without and with adjustment for obesity for greatest clarity.

We acknowledge that it is important to further understand the complex relationships between types of beverages, adiposity, weight change and metabolic risk, and to explore mechanisms underlying associations, and to this end, further research is warranted. As we could not examine the effects of ASB consumption on weight gain or insulin resistance without confounding by obesity status, we echo here the arguments, written by a co-author of this letter (by Sylvetsky Meni et al) that 'it is possible that consumption of artificial sweeteners may be beneficial in limiting weight gain' and 'more studies evaluating the effect of artificial sweeteners on hormonal and metabolic response and on sweet craving must be conducted' [13]. Until we have further evidence otherwise, ASB intake in place of sugary beverages remains a part of weight management strategies. We also further re-endorse our findings that consumption of drinking water and of unsweetened tea or coffee in place of SSB can help in the prevention of type 2 diabetes alongside other healthy lifestyle behaviours.

Acknowledgements We thank M. A. H. Lentjes, Public Health \& Primary Care, University of Cambridge School of Clinical Medicine, for helpful comments.

Funding Work by the authors is supported by a grant from Medical Research Council Epidemiology Unit Core Support (MC_UU_12015/5).

Duality of interest The authors declare that there is no duality of interest associated with this manuscript.
Contribution statement All authors were responsible for drafting the article and revising it critically for important intellectual content. All authors approved the version to be published.

\section{References}

1. Sylvetsky Meni AC, Swithers SE, Rother KI (2015) Positive association between artificially sweetened beverage consumption and incidence of diabetes. Diabetologia doi:10.1007/ s00125-015-3694-5

2. O'Connor L, Imamura F, Lentjes MAH et al (2015) Prospective associations and population impact of sweet beverage intake and type 2 diabetes, and effects of substitutions with alternative beverages. Diabetologia 58:1474-1483

3. Swithers SE, Laboy AF, Clark K et al (2012) Experience with the high-intensity sweetener saccharin impairs glucose homeostasis and GLP-1 release in rats. Behav Brain Res 233:1-14

4. Suez J, Korem T, Zeevi D et al (2014) Artificial sweeteners induce glucose intolerance by altering the gut microbiota. Nature 514:181-186

5. Gardner C, Wylie-Rosett J, Gidding SS et al (2012) Nonnutritive sweeteners: current use and health perspectives: a scientific statement from the American Heart Association and the American Diabetes Association. Circulation 126:509-519

6. Nakagawa Y, Nagasawa M, Yamada S et al (2009) Sweet taste receptor expressed in pancreatic beta-cells activates the calcium and cyclic AMP signaling systems and stimulates insulin secretion. PLoS One 4:e5106

7. Pepino MY, Tiemann CD, Patterson BW et al (2013) Sucralose affects glycemic and hormonal responses to an oral glucose load. Diabetes Care 36:2530-2535

8. Fowler SPG, Williams K, Hazuda HP (2015) Diet soda intake is associated with long-term increases in waist circumference in a biethnic cohort of older adults: the San Antonio Longitudinal Study of Aging. J Am Geriatr Soc 63:708-715

9. Fowler SP, Williams K, Resendez RG et al (2008) Fueling the obesity epidemic? Artificially sweetened beverage use and longterm weight gain. Obesity (Silver Spring) 16:1894-1900

10. Maersk M, Belza A, Stødkilde-Jørgensen H et al (2012) Sucrosesweetened beverages increase fat storage in the liver, muscle, and visceral fat depot: a 6-mo randomized intervention study. Am J Clin Nutr 95:283-289

11. Tate DF, Turner-McGrievy G, Lyons E et al (2012) Replacing caloric beverages with water or diet beverages for weight loss in adults: main results of the Choose Healthy Options Consciously Everyday (CHOICE) randomized clinical trial. Am J Clin Nutr 95:555-563

12. De Ruyter JC, Olthof MR, Seidell JC et al (2012) A trial of sugarfree or sugar-sweetened beverages and body weight in children. N Engl J Med 367:1397-1406

13. Sylvetsky A, Rother KI, Brown R (2011) Artificial sweetener use among children: epidemiology, recommendations, metabolic outcomes, and future directions. Pediatr Clin North Am 58:1467-1480 